\title{
Assessing the Possibility of Presenting a Semi-Stochastic Speed-Density Function
}

\author{
Mohammad Maghrour Zefreh, David Baranyai and Adam Torok \\ Budapest University of Technology and Economics, Department of Transport Technology and Economics, Muegyetem rkp 3, Budapest, \\ Hungary
}

\begin{abstract}
Underlying a fundamental diagram is a relation between traffic speed and density which roughly corresponds to drivers' speed choices under varying car-following distances. Stochastic and deterministic models are mainly two different categories of speed-density models. The advantages of deterministic models are their mathematical simplicity and analytical tractability though their results will show just the average parameters. Although the stochastic models may represent more accurate results taking uncertainty into account, they are often hard to use and analytically not tractable. The aim of this paper is to investigate the possibility of presenting a model which is neither completely deterministic nor completely stochastic but easy to use and understand which incorporates different traffic conditions and speed distributions. Monte Carlo Method has been used to generate different speed distributions based on different traffic conditions and consequently generating their relevant densities. Surveying the relation between the mentioned speed distributions and the obtained densities kept the chance of presenting a model which is neither completely deterministic nor completely stochastic but easy to use and understand which incorporates different traffic conditions and speed distributions.
\end{abstract}

\section{Introduction}

Flying birds, running water, electric current, internet packets, and moving vehicles can all be considered as flows, yet each exhibits distinct characteristics. One of the attributes that distinguishes vehicular traffic flow from other kinds of flow is the so-called fundamental diagram - the relationships among traffic flow characteristics (e.g. flow q, speed v, and density k) which are typically represented graphically [1].

Fundamental relations of traffic flow have historically been established either empirically or derived from carfollowing models. Generally, they come in two flavors: speed-density relations and flow-density relations, which, respectively, express the mean speed and the mean flow rate as functions of traffic density. The speed-density relationship serves as the basis to understand system dynamics in various disciplines [2]. It can be used as a tool to study on moving objects (or particles) in many scientific areas: pedestrians [3, 4], conveyors, network information packages [5], crowd dynamics [6], molecular motors, and biological systems [7]. It has been almost 75 years since Greenshields' seminal paper Study of Traffic Capacity in 1935 [8]. Attaching empirically derived curves to a fitted linear model of the speed-density relationship started a new era of transportation science and engineering. Due to its strong empirical nature, the efforts to find a perfect theory to explain these particular shapes mathematically never cease, but they always achieve limited success. There is a fairly large amount of effort devoted to revising or improving such an oversimplified relationship. These efforts include singleregime models: Greenberg's Model [9], the Underwood Model [10], Northwestern [11], Drew [12], and the PipesMunjal Generalized Model [13].There are also multiregime models which include: two-regime models such as Edie Model [14], multi-regime model by cluster analysis [15], two-regime model [16], modified Greenberg, and three-regime models $[16,11]$.

A generalized deterministic form of speed-density relationship (so-called regression models or fitting models) is written in Eq. 1.

$$
v_{k}=v(k)+\varepsilon
$$

where, $\mathrm{v}(\mathrm{k})$ is the deterministic speed-density relationship in which given density $\mathrm{k}$ there is one fixed corresponding speed value and $\varepsilon$ is the measurement error [2]. Deterministic models are justified on the basis that they describe the average value of the dependent variable given the independent variable inputs [17].

The advantages and drawbacks of deterministic single- and multi- regime speed-density models have been well-documented in a multitude of publications [16, $18,19]$. The advantages of deterministic models are their mathematical simplicity and analytical tractability. The main drawback of deterministic models is that they describe average system behaviors. However, the mean alone may not be capable of describing the entire traffic 
conditions. It should be underlined that traditional deterministic families of models which essentially describe a 'pair-wise' relation between speed and density are not sufficient to include the traffic speed variance.

In the stochastic modeling framework of speeddensity relationship, traffic speed $\mathrm{v}$ is a random function of density $\mathrm{k}$ and random variable $\omega$. In this type of modeling, the random process will be discretized by an appropriate expansion technique which characterizes second-order statistics of the random process using uncorrelated random variables and the deterministic orthogonal set of functions. A simple illustration of the deterministic and stochastic model is shown in Fig. 1.

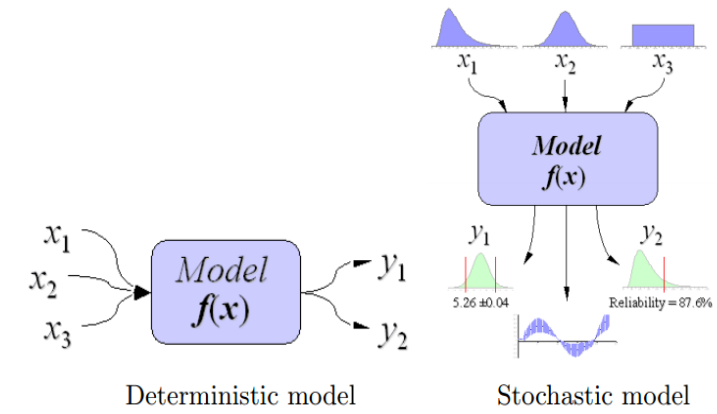

Figure 1. A simple illustration of deterministic and stochastic model [20]

To sum up, stochastic models are hard to use and deterministic models are not enough accurate. The aim of this paper is to investigate the possibility of presenting a model which is neither completely deterministic nor completely stochastic but easy to use and understand which incorporates different traffic conditions and speed distributions. Practically this paper describes the possibility of presenting a model that has deterministic input parameters (desired deterministic density) and stochastic output parameters (related speed distribution) considering different traffic conditions (Referring to Fig. 1.). The main contribution of this paper is to survey on the relation of speed-density parameters to see whether it is possible to derive speed parameter capable of presenting behaviour of traffic dynamics rather than presenting just the average speed of the entire traffic flow.

\section{Methodology}

Since there is a distribution of traffic speed at a certain density level due to the stochastic nature of traffic flow, automatically 'pairwise' pattern from deterministic models will be in contrast with the nature of traffic flow. What if in deterministic speed-density models a certain level of density will be incorporated with one or more part of different speed distributions related to that specific traffic condition?!

The development of mathematical tools focused on the modelling of the speed distribution in a traffic flow is widely reported in the scientific literature [21-24]. Many papers concern this problem since vehicles speed distribution is an important input parameter in lots of issues, such as kinematical traffic simulation model, road design, speed limit evaluation, road traffic noise prediction, traffic safety evaluation, bicycle performance evaluation, analysis of pedestrian walking, road transport emission estimation [25-33], [46] etc. Since 1940 majority of papers considered speed distribution as Gaussian or Normal distribution (Fig. 2) [34] and only extreme situations are considered as the traffic volume exceeds the practical road capacity, the speed distribution may become so heavily skewed toward the higher speeds that all semblance of normality is lost.

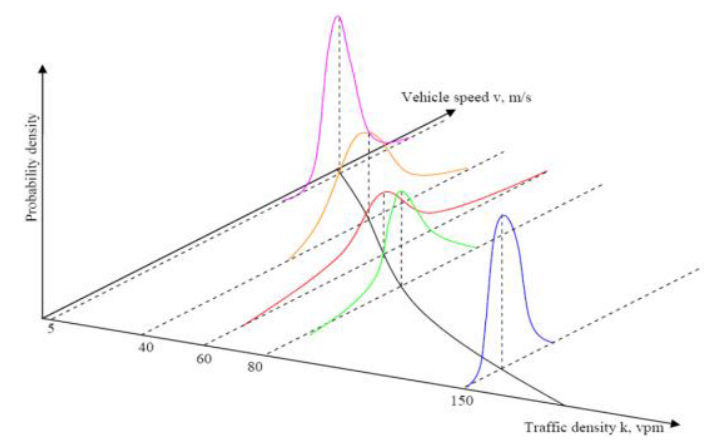

Figure 2. A simple illustration of stochastic speed-density model [2]

If the traffic conditions are not homogeneous, the normal distribution is not well suitable anymore. There is a lot of research that has examined distribution models for motorized vehicle speed data, such as normal distribution [35-37], lognormal distribution [38, 39], skew-t distribution [40, 41], gamma distribution [42], Beta distribution and Chi-Square distribution [25], as well as composite distribution [43]. Overall, roadway sections may have more than two modes, e.g., uncongested speed range, interim speed range which lies between uncongested and congested conditions, and congested speed range. Jun [43] mentioned if the characteristics of speed data are homogeneous (or unimodal distribution), the speed data of interstate freeways can be generally described by conventional probability density function (pdf) such as normal distribution, gamma distribution, lognormal distribution etc. However, if speed data follow a bimodal distribution (or a mixed distribution), there is no specific distribution function available [22].

The conditions that turn to a different speed distribution are quite often realized in non-highway or urban roads, where, in general, the traffic stream is much more complicated. This assumption is supported by Harmonoise [44], where different speed distributions are related to different traffic situations. This large spread of typologies, together with other parameters such as the presence of traffic lights and the road surface conditions, leads to a significant deviation of the speed distribution curve from the generally accepted unimodal normal distribution [25]. In the present research, the traffic condition has been assumed mixed containing the all possible traffic conditions and consequently the all possible speed distributions in order to cover all the possible scenarios.

In the current paper, Monte Carlo Method has been used to build different speed distributions and generate a link between them and their related density. Monte-Carlo methods (or Monte-Carlo simulations) are computational algorithms that rely on repeated random sampling [45]. 
This method generates suitable random numbers of parameters or inputs to explore the behaviour of a complex system or process. The random numbers generated follow a certain probability distribution function (PDF) that describes the occurrence probability of an event. Typically simulations run many times in order to obtain the distribution of an unknown probabilistic parameter. It should be underlined that the number of vehicles, their average speed, and travel distance are remain constant in all cases as similar inputs in Monte Carlo Simulation, but the outputs (speed and density) were totally different based on the nature of traffic flow. Generated speed distributions by Monte Carlo Method have been shown in Fig. 3.

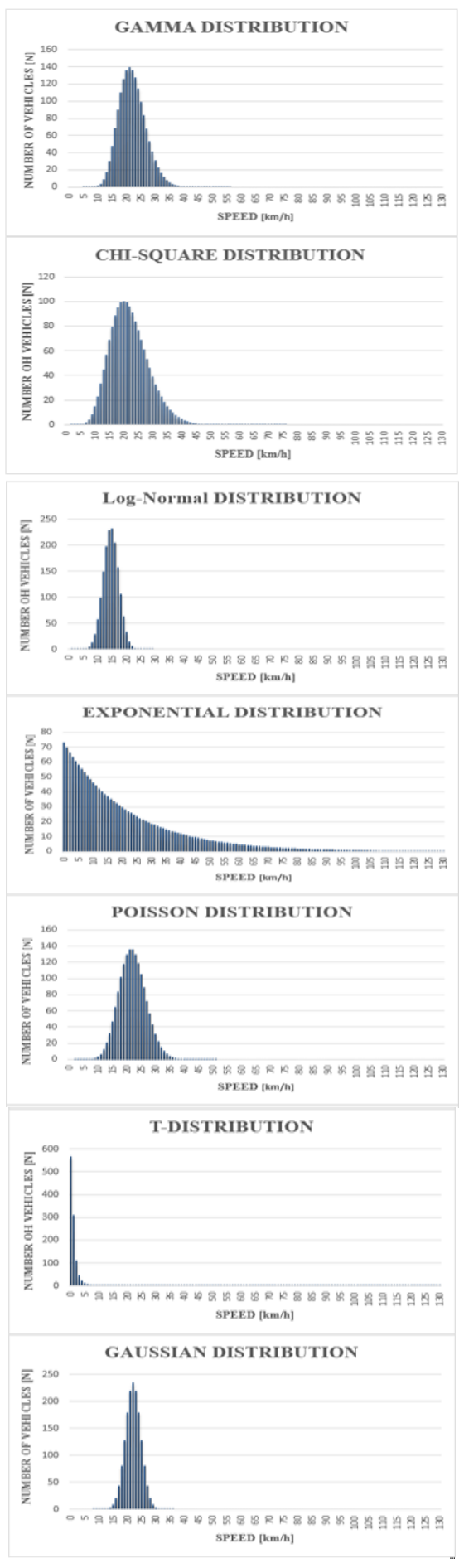

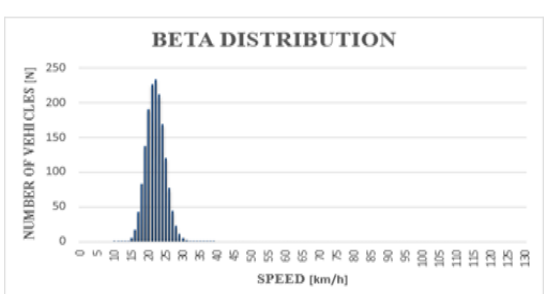

Figure 3. Different speed distributions generated by Monte Carlo Method

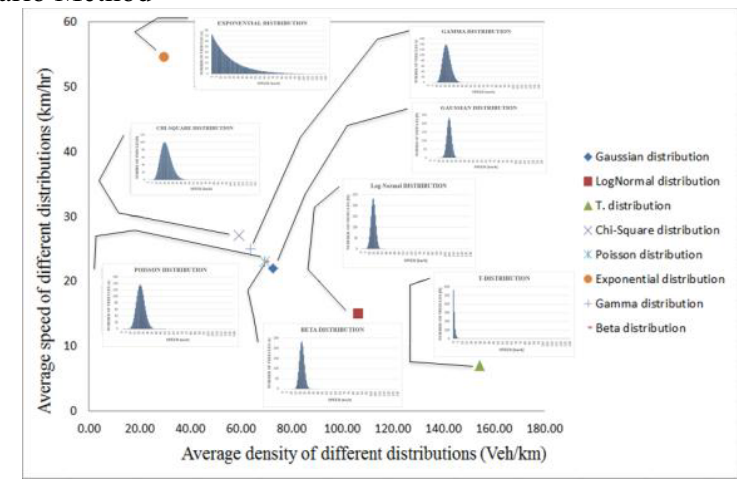

Figure 4. Correlation of the obtained densities and their relevant speed distribution

After running the Monte Carlo Method, generated speed distributions were converted to their relevant densities and the Average and Variance of the obtained density related to each and every speed distribution were computed. These different densities related to different speed distributions (different traffic condition) of the sample highway were shown in Fig. 4.

\section{Results}

By surveying different densities, it was found that in some cases different speed distributions will describe better the traffic conditions and some speed distributions were roughly covered each other (e.g. Poisson and beta distribution). In this paper based on the international literature overview, the different traffic scenarios were identified and described. Schematic fitting of the mentioned correlation in Fig. 4. by generally accepted deterministic speed-density models may imply the possibility of capturing the chance of presenting the semistochastic speed density model which deals with different traffic conditions rather than the average speed (see Fig. 5.).

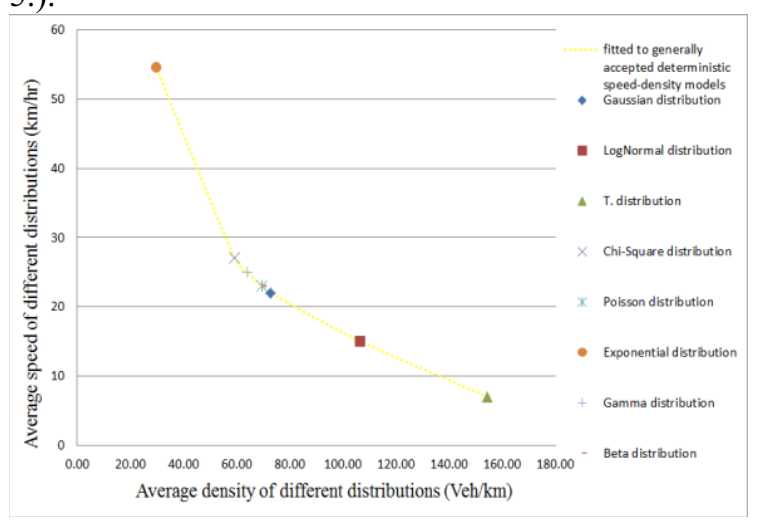

Figure 5. Schematic fitting of the Average speed-density diagram 
It should be highlighted that these schematic fitting (Fig.5.) is based on the relevant average speed and density of different speed distributions. It should be noted that these different speed distributions may have the intersection in some certain densities with each other with different weights. For instance, although the average of the exponential speed distribution is around $55 \mathrm{~km} / \mathrm{hr}$, its domain is between zero $\mathrm{km} / \mathrm{hr}$ and around $110 \mathrm{~km} / \mathrm{hr}$ with more weight toward zero $\mathrm{km} / \mathrm{hr}$ (see Fig.4.). Since these different speed distributions are based on different traffic conditions, this fact implies that the output of the deterministic speed-density function cannot be a single average speed but should be the function of speed composed of different relevant speed distributions.

\section{Conclusion}

In this paper, the authors tried to provide a method in which traffic speed variance will be incorporated by traffic density. The aim of this paper was to investigate the possibility of presenting a model which is neither completely deterministic nor completely stochastic but easy to use and understand and incorporates different traffic conditions by determining different speed distributions. The main contribution of this paper is to survey on the relation of speed-density parameters to see whether it is possible to derive speed parameter capable of presenting behaviour of traffic dynamics rather than presenting just the average speed of the entire traffic flow. The results showed that different speed distribution based on different traffic conditions may have the intersection with each other- however their averages were fitted to the generally accepted deterministic speed-density modelsand this fact implies that discrete modelling is not viable in this case. The scope for future research of the authors will be applying the fuzzy theory to help the composition of the complex speed-density probability function taking intersections into account. The final complex semistochastic model will be validated by the traffic data of Budapest, Hungary compared to other density-velocity models.

\section{References}

1. Wang, Haizhong, Jia Li, Qian-Yong Chen, and Daiheng Ni. 2011. Transportation Research Part A: Policy and Practice 45 (6): 554-66. doi:10.1016/j.tra.2011.03.010

2. Wang, Haizhong, Jia Li, Qian-Yong Chen, and Daiheng Ni. 2009. The 88th Transportation Research Board (TRB) Annual Meeting. Washington, DC. http://people.umass.edu/ndh/Publications/C21.pdf

3. Jr., S. J. Y. Smith, J. M. Modeling Circulation Systems in Buildings Using State Dependent Queueing Models,Queueing Syst., 1989, 4, pages: 319-338.

4. Rajat, J. Smith, J. Modeling Vehicular Traffic Flow using $\mathrm{M} / \mathrm{G} / \mathrm{C} / \mathrm{C}$ State Dependent Queueing Models. Transportation Science, 31, pages: 324-335, (1997).
5. Gabor S, C. I. The analogies of highway and computer network traffic. Physica A, 2002, 307, pages: 516-526.

6. Helbing, D. Traffic and Related Self-Driven ManyParticle Systems. Rev. Mod. Phys., 2001,73,10671141.

7. Chowdhury, D. Schadschneider, A., Nishinari, K. Physics of Transport and Traffic Phenomena in Biology: from molecular motors and cells to organisms. Physics of Life Reviews, (2005), 2, 318.

8. Greenshields, B. D. A Study in Highway Capacity. Highway Research Board Proceedings, 1935, 14, pages: 448-477.

9. Greenberg, H. An analysis of traffic flow. Operation Research, (1959), 7, pages: 79-85.

10. Underwood, R. T. Speed, Volume, and Density Relationship. Quality and Theory of Traffic Flow, Yale Bur. Highway Traffic, New Haven, Connecticut, (1961), pages: 141-188.

11. J. S. Drake, J. L. Schofer, A. D. May. A Statistical Analysis of Speed Density Hypotheses. Third International Symposium on the Theory of Traffic Flow Proceedings, Elsevier North Holland, Inc. New York, (1967).

12. Drew, D. R. Traffic Flow Theory and Control, McGraw-Hill Book Company, 1968, Chapter 12.

13. Pipes, L. A. Car-Following Models and the Fundamental Diagram of Road Traffic. Transportation Research, 1967, 1, pages: 21-29.

14. Edie, L. C. Car-Following and Steady-State Theory for Noncongested Traffic. Operation Research, (1961), 9, pages: 66-76.

15. Lu Sun, J. Z. Development of Multiregime SpeedDensity Relationship by Cluster Analysis. Transportation Research Record, (2005), Volume 1934, pages: 64-71.

16. May, A. D. Traffic Flow Fundamentals, Prentice Hall, Englewood Cliffs, (1990).

17. Douglas I. Rouse. Stochastic Modeling of Plant Disease Epidemic Processes, Handbook of Applied Mycology Sail and Plants, Vol. 1 edited by Dilip K. Arora etc, (1991).

18. Hall, F.L., Hurdle, V.F., Banks, J.H. A synthesis on recent work on the nature of speed flow and flowoccupancy (or density) relationships on freeways. Transportation Res. Rec. 1365, pp. 12-18, (1992).

19. James H. Banks. Review of Empirical Research on Congested Freeway Flow. Transportation Res. Rec. 1802, pp. 225-232, (2002).

20. J.W. Wittwer, 'Monte Carlo Simulation Basics ', 2004. [Online]. Available: http://www.vertex42.com/ExcelArticles/mc/MonteC arloSimulation.html. [Accessed: 03- March- 2016].

21. Castro M., Sanchez J.A., Vaquero C.M., Iglesias L., Rodriguez-Solano R., (2008), Automated GIS-based system for speed estimation and highway safety evaluation, Journal of Computing in Civil Engineering, 22, 325-331.

22. Dey P.P., Chandra S., Gangopadhaya S., (2006), Speed distribution curves under mixed traffic conditions, Journal of transportation engineering, $132,475-481$. 
23. Fitzpatrick K., Carlson P.L., Wooldridge M.D., Brewer M.A., (2000), Design factors that affect driver speed on suburban arterials, Technical Report Documentation, Texas Transportation Institute, Report n. 1769-3.

24. Trozzi C., Vaccaro R., Crocetti S., (1996), Speed frequency distribution in air pollutants emissions estimate from road traffic, Science of the Total Environment, 189/190, 181 - 185.

25. Iannone, G., Guarnaccia, C., \& Quartieri, J. (2013). Speed distribution influence in road traffic noise prediction. Environmental Engineering And Management Journal, 12(3), 493-501.

26. Berry, Donald S., Daniel M. Belmont, and others. 1951. "Distribution of Vehicle Speeds and Travel Times." In Proceedings of the Second Berkeley Symposium on Mathematical Statistics and Probability, 589-602. The Regents of the University of California. http://digitalassets.lib.berkeley.edu/math/ucb/text/ma th_s2_article-43.pdf.

27. Lin S, He M, Tan Y, et al. Comparison study on operating speeds of electric bicycles and bicycles: experience from field investigation in Kunming, China. Transp. Res. Record (2008); 2048: 52-59.

28. Wang D, Zhou D, Jin S, et al. Characteristics of mixed bicycle traffic flow on conventional bicycle path. Presented at 94th annual meeting of the transportation research board, Washington, DC, 1115 January (2015).

29. Vadeby, Anna, and \AAsa Forsman. 2014. Transport Research Arena (TRA) 5th Conference: Transport Solutions from Research to Deployment. http://tra2014.traconference.eu/papers/pdfs/TRA201 4 _Fpaper_18042.pdf.

30. Maurya, A.K., Das, S., Dey, S. (2015), 11th International Conference of Eastern Asia Society for Transportation Studies (EASTS 2015) in Cebu City, Philippines during September 11-14, 2015.

31. Hustim, M. Fujimoto, K. (2012) Road Traffic Noise under Heterogeneous Traffic Condition in Makassar City, Indonesia. Journal of Habitat Engineering and Design, 4(1), 109-118.

32. B., Aly, S.H., Ramli, M.I, Sumi, T. (2012) Driving Cycle of Passenger Cars on Heterogeneous Traffic Situations: Case Study on an Urban Road in Makassar, Indonesia. Proceeding of the 8th International Symposium on Lowland Technology.

33. Chandra, Satish, and Anish Kumar Bharti. 2013. "Speed Distribution Curves for Pedestrians During Walking and Crossing." Procedia - Social and Behavioral Sciences 104 (December): 660-67. doi:10.1016/j.sbspro.2013.11.160.
34. Loutzenheiser, D. W., \& Greenshields, B. D. (1940) Percentile Speeds on Existing Highway Tangents. In Highway Research Board Proceedings (Vol. 20).

35. Leong HJW. The distribution and trend of free speeds on two-lane two-way rural highways in New South Wales. In: Proceedings of the 4th Australian road research board conference, part 1, Melbourne, VIC, Australia, 14-16 October 1968, pp.791-808. Vermont South, VIC, Australia: Australian Road Research Board.

36. McLean J. R. (1978): Observed Speed Distributions and Rural Road Traffic Operations. 9th Australian Road Research Board Conference Proceedings., Part 5, Australian Road Research Board, Vermont South, Victoria, Australia, 235-244.

37. Minh, C. C., Sano, K., and Matsumoto, S. (2005) The speed, Flow and Headway Analyses of Motorcucle Traffic. Journal of the Eastern Asia Society for Transportation Studies, 6, 1496-1508.

38. Wang, Y., Dong, W., Zhang, L., Chin, D., Papageorgiou, M., Rose, G., Young, W. (2012) Speed modeling and travel time estimation based on truncated normal and lognormal distributions. Journal of the Transportation Research Board, No. 2315, 66-72.

39. Gerlough DL and Huber MJ. Traffic flow theory: a monograph. Special report 165 . Washington, DC: Transportation Research Board, National Research Council, June, 1975.

40. Zou, Y. (2013) A Multivariate Analysis of Freeway Speed and Headway Data. Texas A\&M Transportation Institute.

41. Zou, Y., and Zhang, Y. (2011) Use of Skew-Normal and Skew-t Distributions for Mixture Modeling of Freeway Speed Data. Journal of the Transportation Research Board, No. 2260, 67-75.

42. Haight F.A., Mosher W.W., (1962), A practical method of improving the accuracy of vehicular speed distribution measurements, HRR 341, Highway Research Board, Washington, D.C., 92116.

43. JUN, J.. Understanding the variability of speed distributions under mixed traffic conditions caused by holiday traffic. Transportation Research Part CEmerging Technologies, 18, 599-610, (2010).

44. Harmonoise. (2004), Imagine project funded by EC under the sixth framework program, On line at: http://www.imagine-project.org.

45. Torok, Adam. 2015. International Journal for Traffic and Transport Engineering 5 (3): 278-85. doi:10.7708/ijtte.2015.5(3).05.

46. A. Török and M. M. Zefreh, Acta Tech. Jaurinensis, 9, no. 2, p. 118, Apr. (2016) 